\title{
Wearable Piezoelectric-Based System for Continuous Beat-to-Beat Blood Pressure Measurement
}

\author{
Ting-Wei Wang $1(\mathbb{C}$ and Shien-Fong Lin $1,2,3, * \mathbb{1}$ \\ 1 Department of Electrical and Computer Engineering, College of Electrical and Computer Engineering, \\ National Chiao Tung University, Hsinchu 30010, Taiwan; w756704@gmail.com \\ 2 Institute of Biomedical Engineering, College of Electrical and Computer Engineering, National Chiao Tung \\ University, Hsinchu 30010, Taiwan \\ 3 Krannert Institute of Cardiology, Indiana University School of Medicine, Indianapolis, IN 46202, USA \\ * Correspondence: linsf5402@nctu.edu.tw
}

Received: 16 January 2020; Accepted: 4 February 2020; Published: 5 February 2020

\begin{abstract}
Non-invasive continuous blood pressure measurement is an emerging issue that potentially can be applied to cardiovascular disease monitoring and prediction. Recently, many groups have proposed the pulse transition time (PTT) method to estimate blood pressure for long-term monitoring. However, the PTT-based methods for blood pressure estimation are limited by non-specific estimation models and require multiple calibrations. This study aims to develop a low-cost wearable piezoelectric-based system for continuous beat-to-beat blood pressure measurement. The pressure change in the radial artery was extracted by systolic and diastolic feature points in pressure pulse wave (PPW) and the pressure sensitivity of the sensor. The proposed system showed a reliable accuracy of systolic blood pressure (SBP) (mean absolute error (MAE) \pm standard deviation (SD) $1.52 \pm 0.30 \mathrm{mmHg}$ ) and diastolic blood pressure (DBP, MAE \pm SD $1.83 \pm 0.50)$, and its performance agreed with standard criteria of MAE within $5 \mathrm{mmHg}$ and SD within $\pm 8 \mathrm{mmHg}$. In conclusion, this study successfully developed a low-cost, high-accuracy piezoelectric-based system for continuous beat-to-beat SBP and DBP measurement without multiple calibrations and complex regression analysis. The system is potentially suitable for continuous, long-term blood pressure-monitoring applications.
\end{abstract}

Keywords: continuous blood pressure; piezoelectric sensor; wearable device

\section{Introduction}

Blood pressure measurement is an essential technique used in cardiovascular disease monitoring. The auscultatory and oscillometric methods, which use the cuff sensor to detect systolic blood pressure (SBP) and diastolic blood pressure (DBP), are the golden benchmark and commonly used in clinical diagnosis. However, the cuff-based technique only provides one-shot data without continuous blood pressure information and causes inconvenience due to repeated cuff inflations. Therefore, a significant limitation of the cuff-based approach is the lack of continuous monitoring of adverse events for hypertensive patients. On the other hand, the arterial cannula is a precise method to directly measure continuous blood pressure, but the arterial cannula method uses a catheter inserted into the blood vessel that could induce potential risk by such an invasive method [1]. Consequently, the non-invasive continuous blood pressure monitoring remains a significant clinical unmet need.

Many groups have proposed methods based on pulse transition time (PTT) for continuous blood pressure monitoring [2-4]. PTT represents the estimated propagation time of a pulse wave measured between two sites of the artery [5,6]. PTT is frequently extracted from two-channel physiological signals, including electrocardiography (ECG) and photoplethysmography (PPG). PTT is determined by the time delay between the R-peak of the ECG signal and the time point of the maximum slope 
of the PPG waveform $[7,8]$. The PTT can also be estimated from two-channel PPG signals recorded from two different locations to calculate the time delay between both waveforms $[9,10]$. Many studies presented several PTT-based mathematical models for blood pressure estimation by different models [11], including logarithmic model [12], linear model [13], inverse square model [14], and inverse model [15]. The regression coefficients of these models were obtained by multiple calibrations between the oscillometric method (the gold standard) and PPT-based technique to determine the accuracy of the model. At present, the application of PTT-based methods is limited by technical considerations, including the appropriate two-channel physiological measurement, non-specific estimation models and a complex procedure of multiple calibrations.

In order to deploy a wearable device with a one-channel physiological measurement, some studies provided a pressure sensor to achieve continuous blood pressure monitoring. The pulsation changes in the artery can be detected by a pressure sensor such as the capacitive pressure sensor and piezoelectric sensor, which were usually used in blood pressure applications depending on the pressure sensitivity of the device. The capacitive pressure sensor uses the mechanism of distance change between two parallel plates to estimate blood pressure change in the artery. However, capacitive pressure sensors are typically characterized by low-pressure sensitivities due to the small stress from arterial compression. Kim et al. [16] presented a modified structure of the dielectric layer by PDMS spacer and wrinkle gold foil to improve the sensitivity of the capacitive pressure sensor for blood pressure measurement application. Compared to the capacitive pressure sensor, a piezoelectric sensor directly converts the pressure signals into electrical signals by pressure sensitivity $(\mathrm{mV} / \mathrm{mmHg})$ of the piezoelectric sensor. The radial artery of the wrist is the common site to measure continuous pressure pulse wave (PPW) signals by a piezoelectric sensor. For example, Liu et al. [17] demonstrated a PPW-based blood pressure estimation models by piezoelectric sensor implementation and a linear regression method to demonstrate the low mean absolute error (MAE) of blood pressure measurement, compared to the cuff method. Recently, Kaisti et al. [18] developed a wearable microelectromechanical system (MEMS) that produced a high correlation of mean arterial pressure (MAP) between the non-invasive pulse measurement and the invasive pulse waveform.

Most of these previous studies sought to increase accuracy through complicated mathematical models and calculations. Alternatively, an intuitive computation and reliable accuracy are preferable for practical applications in continuous beat-to-beat blood-pressure measurement. The main aim of this paper is to develop a wearable piezoelectric-based system to provide an intuitive strategy that uses the summation of initial blood pressure and pressure change for beat-to-beat SBP and DBP measurement without the cumbersome multiple calibrations.

\section{Measurement Strategy}

The measurement strategy uses the sum of the initial blood pressure measured by a cuff-based sensor (HEM-1000, OMRON) with oscillmotric method and pressure changes obtained by a calibrated piezoelectric sensor to achieve beat-to-beat SBP and DBP monitoring, according to Equation (1).

$$
P(t)=P_{\text {initial }}+\Delta P
$$

The changes in the pressure of the artery can be calculated by the pressure sensitivity of the piezoelectric sensor. The step-by-step procedure of the measurement strategy is the following:

Step 1: The initial blood pressure is measured by the cuff method.

Step 2: The pressure signals from the radial artery are converted to an electrical signal through the piezoelectric sensor. Continuous PPW signals are filtered and amplified by the front-end analog circuit

Step 3: The voltage change of SBP and DBP feature points is identified within the adjacent PPW signals (Figure 1a). 
Step 4: The voltage change $(\triangle \mathrm{mV})$ of SBP and DBP feature points are converted to pressure change $(\triangle \mathrm{mmHg}$ ) of SBP and DBP by the pressure sensitivity of the piezoelectric sensor (Figure $1 \mathrm{~b}$ ).

Step 5: Sum of initial blood pressure by cuff method and pressure changes by the piezoelectric sensor to achieve beat-to-beat SBP and DBP monitoring, according to Equations (2) and (3) (Figure 2)

$$
\begin{aligned}
& S B P(t)=S B P_{c u f f, \text { initial }}+\Delta m m H g_{1}+\Delta m m H g_{2}+\ldots \\
& D B P(t)=D B P_{c u f f, \text { initial }}+\Delta m m H g_{a}+\Delta m m H g_{b}+\ldots
\end{aligned}
$$

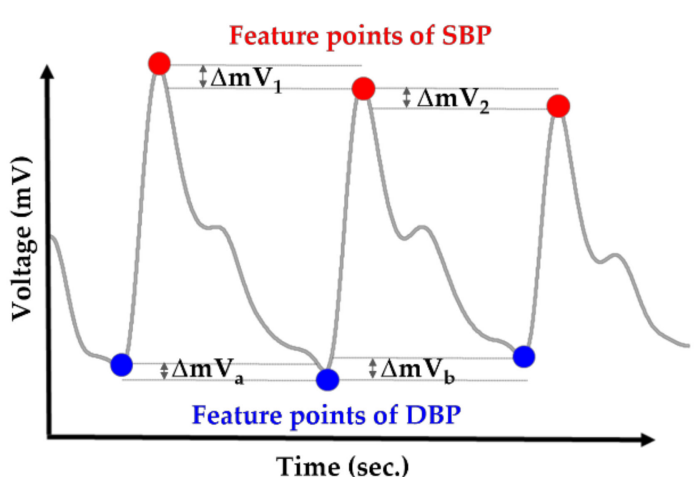

(a)

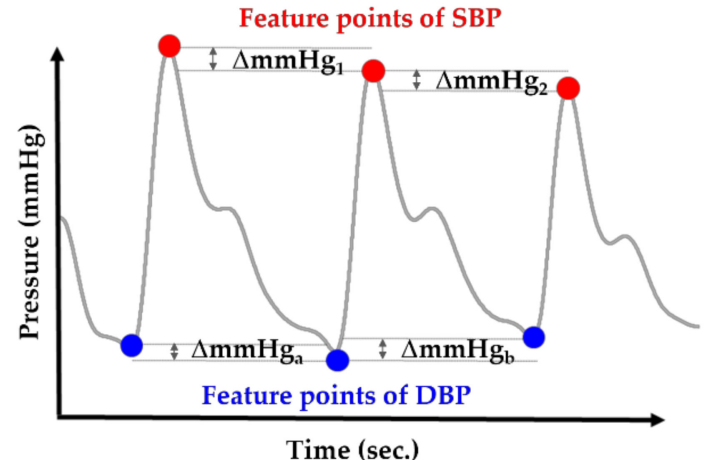

(b)

Figure 1. (a) Calculate the $\Delta \mathrm{mV}$ at the systolic blood pressure (SBP) and diastolic blood pressure (DBP) feature points within the adjacent wave. (b) Convert the voltage change $(\Delta \mathrm{mV})$ into pressure change $(\Delta \mathrm{mmHg})$ by the pressure sensitivity of the piezoelectric sensor $(\mathrm{mV} / \mathrm{mmHg})$.

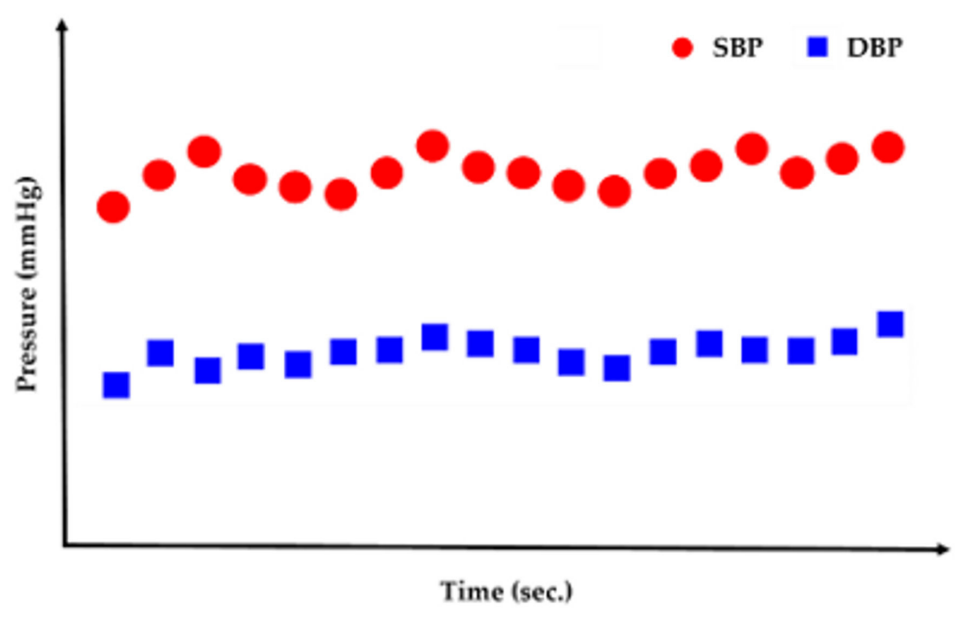

Figure 2. Continuous beat-to-beat SBP and DBP monitoring.

\section{System Design}

The system can be described in three main parts, including piezoelectric sensor, front-end analog circuit, and software processing unit (Figure 3). The piezoelectric sensor provides a sensing function of pulsation changes of the radial artery that converts pressure signals into electrical signals by the pressure sensitivity of the piezoelectric sensor. The front-end analog circuit is responsible for PPW amplification and filtering. The round piezoelectric sensor and the analog frontend circuit were constructed with geometric dimensions of $2.4 \mathrm{~cm}$ in diameter and $5 \mathrm{~cm} \times 7 \mathrm{~cm}$, respectively. The post-processing unit is responsible for detecting SBP and DBP feature points and calculating pressure changes between the adjacent waves. 


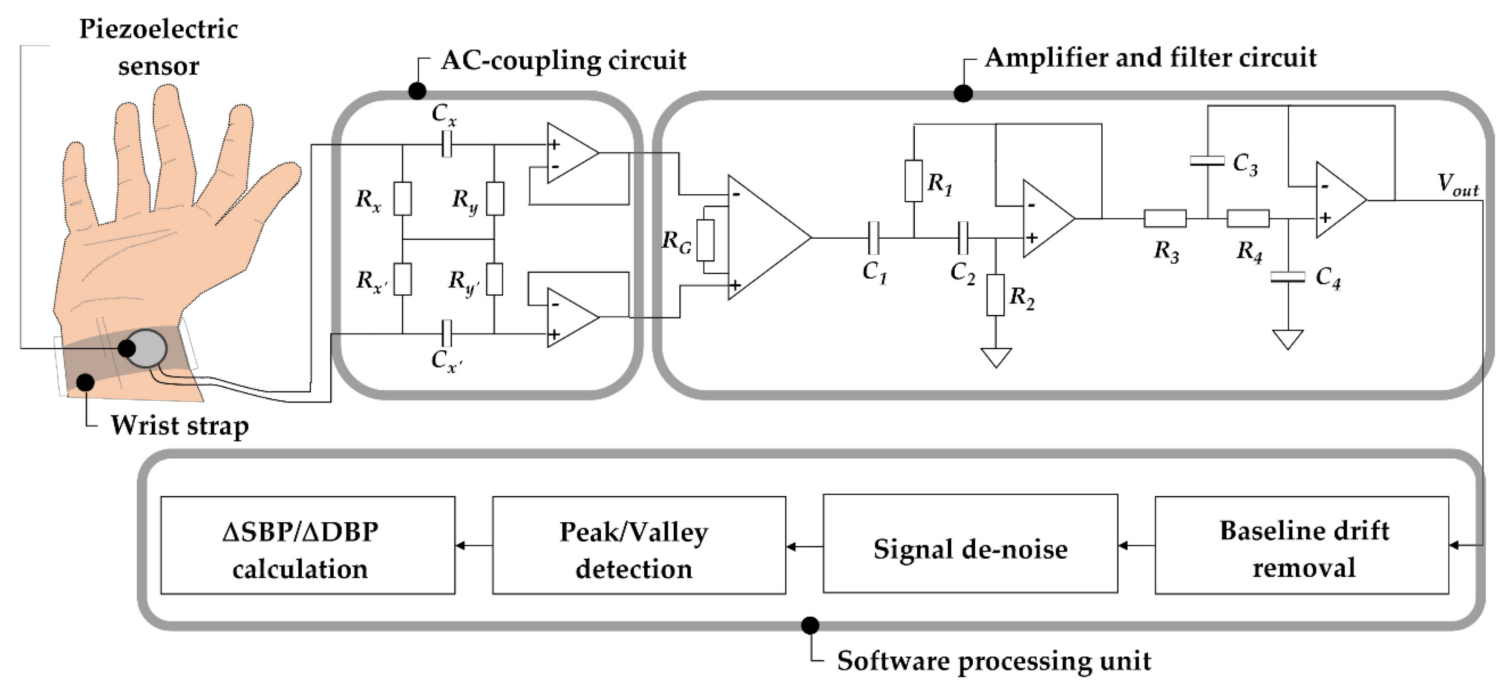

Figure 3. Schematic of the overall system.

\subsection{Piezoelectric Sensor}

The pulsation signal from the radial artery is a weak signal, which is highly susceptible to interference from electric and magnetic fields during the detection procedure. Therefore, the sensitivity, resolution, and anti-interference ability of the sensor must be considered when detecting the PPW signals. A piezoelectric module HK-2000B (Hefei Huake Electronic Technology Research Institute, Hefei, China) was used in this study. The HK-2000B is a medical pulse sensor that uses piezoelectric-sensitive materials of polyvinylidene fluoride (PVDF) film, which directly converts the arterial pressure signals into electrical signals. The main characteristics of HK-2000B are high sensitivity $(2 \mathrm{mV} / \mathrm{mmHg})$ and wide pressure detection range $(-50-3000 \mathrm{mmHg})$. The piezoelectric sensor was mounted on the subject's wrist above the radial artery by a wrist strap, which can maintain stable mechanical coupling of the sensor with the skin (Figure 4).

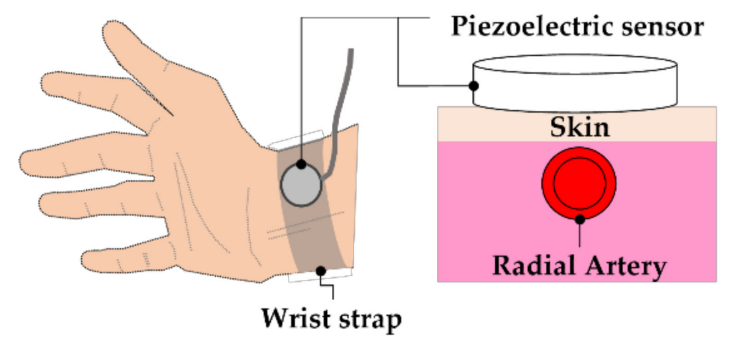

Figure 4. Piezoelectric sensor measurement location.

\subsection{Front-End Analog Circuit}

In order to extract clear PPW signals, a front-end analog circuit is required to process signals obtained from the piezoelectric sensor. The front-end analog circuit can be divided into two parts, including the alternating current (AC)-coupling circuit and amplifier circuit. The AC-coupling circuit is responsible for direct current (DC) removal in bio-potential measurements [19] that provides a differential high-pass filter without the grounded resistors to eliminate baseline wandering in front of the amplifier circuit [20]. The AC-coupling circuit was designed with a cutoff frequency of 0.03 $\mathrm{Hz}$, according to Equation (4). In addition, the high impedance voltage followers were behind the AC-coupling circuit to avoid the signals to attenuate.

$$
f_{c}=\frac{1}{2 \pi R_{y} C_{x}}
$$


AD620 instrumentation amplifiers (Analog Devices Inc., Norwood, MA, USA) were used for signal amplification with optimal power supply of $\pm 15 \mathrm{~V}$ that was provided by the data acquisition (DAQ) device with \pm 15 V DC power source. The AD620 provides a tunable gain of 1 to 10,000 by an external resistor, according to Equation (5). This study chose the gain of 989 using RG of $50 \Omega$ with a high common-mode rejection ratio (CMRR) of $140 \mathrm{~dB}$. In order to cover PPW signals bandwidth, a combination of a Butterworth high-pass filter (HPF) of $0.05 \mathrm{~Hz}$ and low pass filter (LPF) of $35 \mathrm{~Hz}$ were implemented, according to Equations (6) and (7). Based on the analysis of hardware functionality, sweeping frequencies from $10^{-3} \mathrm{~Hz}$ to $10^{3} \mathrm{~Hz}$ were performed to evaluate the frequency response of the front-end analog circuit. The simulation result performed the required bandwidth $(0.05-35 \mathrm{~Hz})$ with an amplification factor of about $59.9 \mathrm{~dB}$ and obtained a lower cutoff frequency $\left(f_{L}\right)$ of $0.05 \mathrm{~Hz}$ and a higher cutoff frequency $\left(f_{H}\right)$ of $35.12 \mathrm{~Hz}$ (Figure 5).

$$
\begin{gathered}
\text { Gain }=\frac{49.4 K \Omega}{\mathrm{R}_{\mathrm{G}}}+1 \\
\mathrm{f}_{\mathrm{HPF}}=\frac{1}{2 \pi \sqrt{\mathrm{R}_{1} \mathrm{R}_{2} \mathrm{C}_{1} \mathrm{C}_{2}}} \\
\mathrm{f}_{\mathrm{LPF}}=\frac{1}{2 \pi \sqrt{\mathrm{R}_{3} \mathrm{R}_{4} \mathrm{C}_{3} \mathrm{C}_{4}}}
\end{gathered}
$$

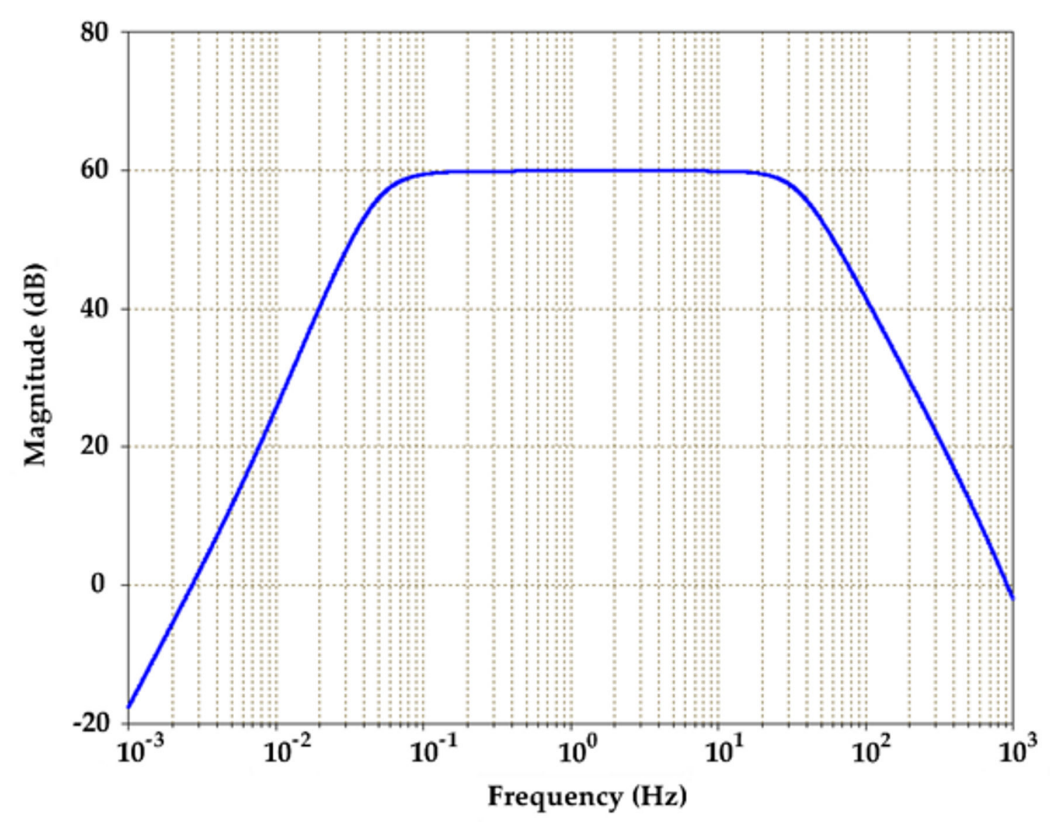

Figure 5. The simulated frequency response of the system.

\subsection{Post-Processing Unit}

A custom-designed software based on the LabVIEW platform was developed to analyze the PPW signal in the post-processing unit. To extract the signals of the arterial pressure pulse, the respiration signal removal was required to eliminate the baseline wandering by detrending the raw recording. The signal denoise was important for peak and valley detection algorithm; therefore, the WA Denoise VI function was implemented after the baseline wandering removal. In order to extract the peak and valley points in PPW signals, a threshold detection method was provided (Figure 6). The voltage information in SBP and DBP feature points was described (the solid lines are de-noise PPW signals, and dashed lines are detection thresholds). The voltage change of SBP and DBP feature points within adjacent PPW signals can be acquired, according to Equations (8) and (9). The pressure sensitivity of the piezoelectric sensor offers a unit conversion of $2 \mathrm{mmV} / \mathrm{mmHg}$ that implies that the pressure 
change is $0.5 \mathrm{mmHg}$ per $\mathrm{mV}$, according to Equation (10). Therefore, the pressure change of SBP and DBP feature points between the adjacent PPW signals were obtained by (11).

$$
\begin{gathered}
\Delta m V_{1}=V_{\max , 2}-V_{\max , 1}, \Delta m V_{2}=V_{\max , 3}-V_{\max , 2}, \ldots \\
\Delta m V_{a}=V_{\min , 2}-V_{\min , 1}, \Delta m V_{b}=V_{\min , 3}-V_{\min , 2}, \ldots \\
\text { Pressure sensitivity }=\frac{2 \mathrm{mV}}{\mathrm{mmHg}} \\
\Delta \mathrm{mmHg}=\frac{\Delta \mathrm{V} \times 1000}{\text { Gain }} \times(\text { Pressure sensitivity })^{-1}
\end{gathered}
$$

\subsection{Ethics Statement}

The experiment was approved by the Institutional Review Board of National Chiao Tung University (registration number: NCTU-REC-108-087E). Thirty subjects participated in the experiment (19 males and 11 females, age $20-60$ years, height $150-183 \mathrm{~cm}$, weight $50-110 \mathrm{~kg}$ ). They were healthy without any known diseases and provided their written informed consent. They were asked to refrain from alcohol, caffeine, and strenuous exercise for $1 \mathrm{~h}$ before the measurement. In the experiment, all subjects consented to participate and were instructed to remain in a sitting position without movement to avoid motion artifacts during the measurement.

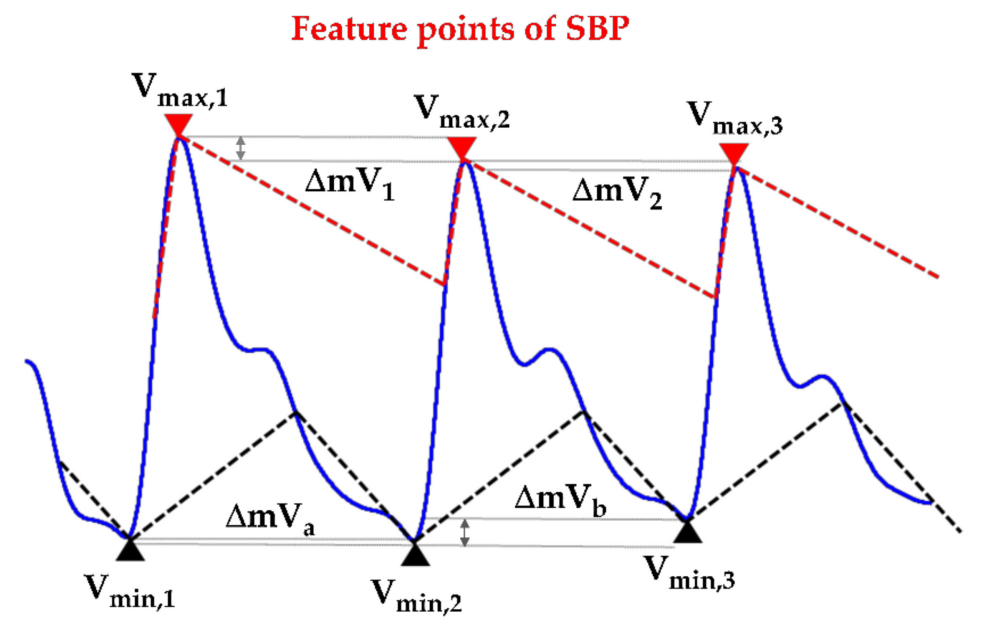

Figure 6. Peak and valley detection algorithm for pressure pulse wave (PPW) signals.

\section{Experimental results}

4.1. Pressure Pulse Wave (PPW) Signals Analysis for Beat-to-Beat Systolic Blood Pressure (SBP) and Diastolic Blood Pressure (DBP)

The piezoelectric sensor was placed on the skin above the radial artery and measured for $10 \mathrm{sec}$ to obtain the continuous pressure waveform. Figure 7a shows the continuous PPW signals obtained from the analog circuit. In order to detect the exact locations of the peak and valley of PPW signals, post-processing was performed to produce distinguishable feature points of SBP and DBP, as shown in Figure $7 \mathrm{~b}$. Figure 8 shows that the peak and valley values were extracted from the continuous PPW post-processing signals by the threshold method. Figure 9a indicates that the voltage change within adjacent PPWs was calculated, according to Equations (8) and (9). The pressure change within adjacent PPWs was obtained by conversion of pressure sensitivity $(2 \mathrm{mV} / \mathrm{mmHg})$ and circuit amplification gain of 989, according to Equation (11). Therefore, the pressure change between adjacent beats can be calculated, as shown in Figure $9 \mathrm{~b}$. The measurement strategy uses the sum of the initial blood pressure by an oscillometric method and pressure change by a piezoelectric sensor to achieve the 
beat-to-beat SBP and DBP monitoring, according to Equation (1). The initial SBP and DBP measured by the cuff-method were respectively $109 \mathrm{mmHg}$ and $61 \mathrm{mmHg}$. Figure 10 demonstrates the beat-to-beat SBP and DBP monitoring.

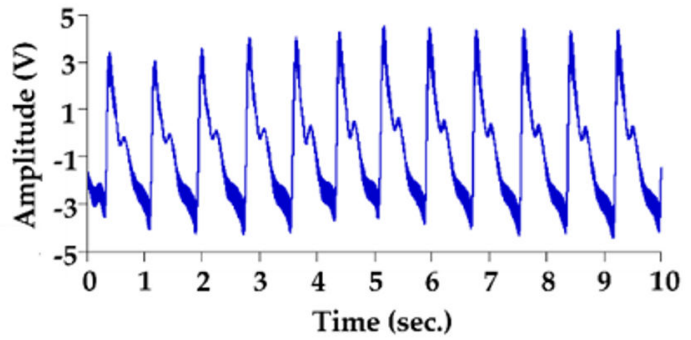

(a)

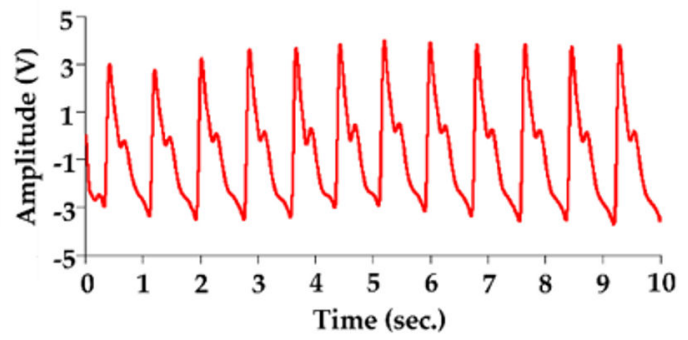

(b)

Figure 7. PPW signals from the (a) analog circuit (b) post-processing unit.

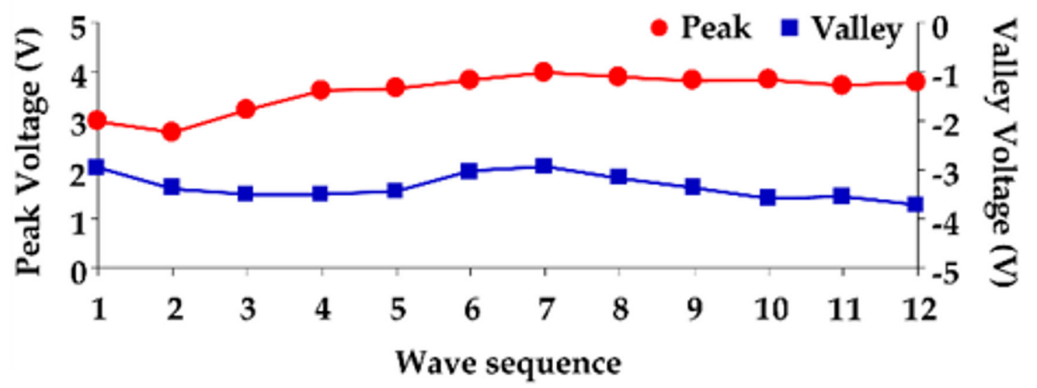

Figure 8. Peak and valley detection algorithm for PPW signals.

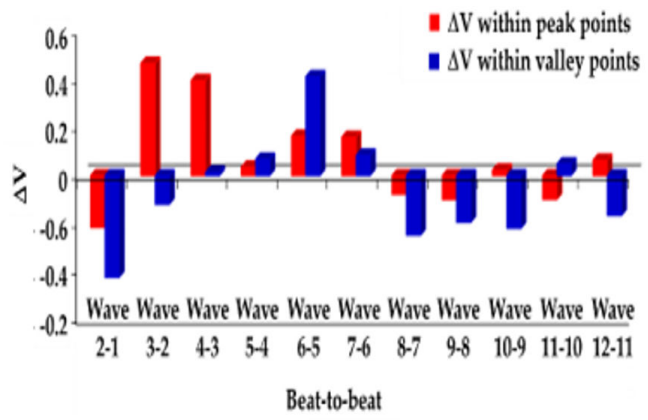

(a)

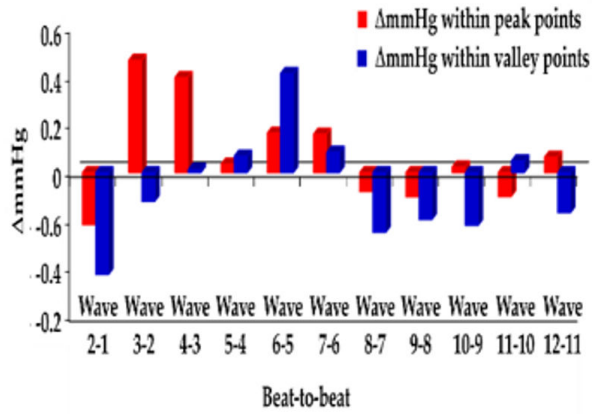

(b)

Figure 9. (a) The voltage change and (b) pressure change within feature points of SBP and DBP. 


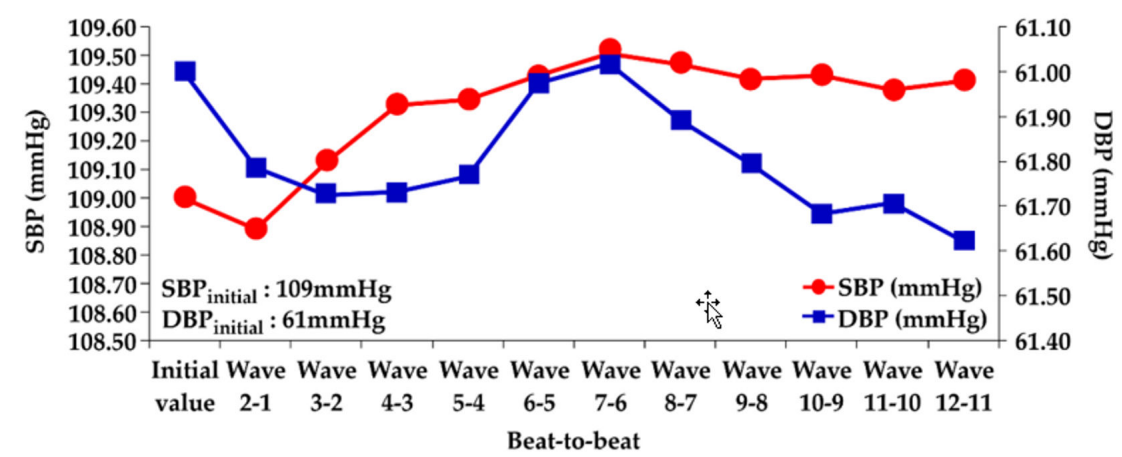

Figure 10. Beat-to beat blood pressure for 12 beats.

\subsection{Continuous Blood Pressure Measurement and Accuracy Evaluation}

In order to perform accuracy evaluation compared to the oscillometric method, the left and right hands of 30 subjects with the individual sensors were synchronously measured for $30 \mathrm{~min}$. The piezoelectric sensor was placed on the left hand to measure the continuously beat-to-beat pressure change for $30 \mathrm{~min}$. The oscillometric sensor was measured on the right hand at a regular interval of $1 \mathrm{~min}$ for 30 measurements in $30 \mathrm{~min}$ (Figure 11). The performance of our system was evaluated by recording the MAE and standard deviation (SD) within two methods, according to Equations (12) and (13).

$$
\begin{gathered}
\text { MAE }=\frac{1}{n} \sum_{i=1}^{n}\left|y_{i}-x_{i}\right| \\
S D=\sqrt{\frac{\sum_{i=1}^{n}\left(y_{i}-x_{i}-M A E\right)^{2}}{n-1}}
\end{gathered}
$$

where $x i$ is the reference blood pressure values obtained from the oscillometric sensor and $y i$ is the measurement blood pressure values obtained from our system on $n$ measurements. Table 1 demonstrates the average MAE \pm SD of SBP and DBP were $1.52 \pm 0.30 \mathrm{mmHg}$ and $1.83 \pm 0.50 \mathrm{mmHg}$ for 30 subjects.

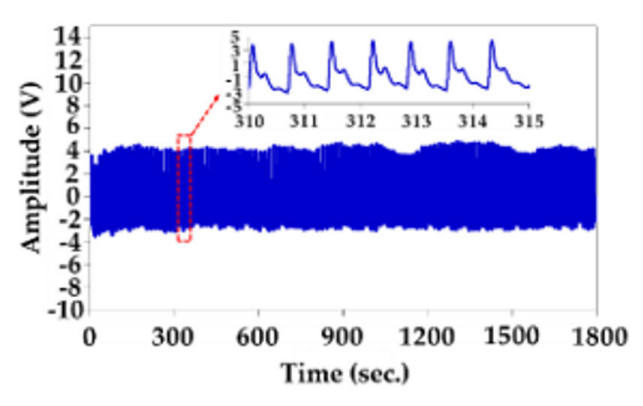

(a)

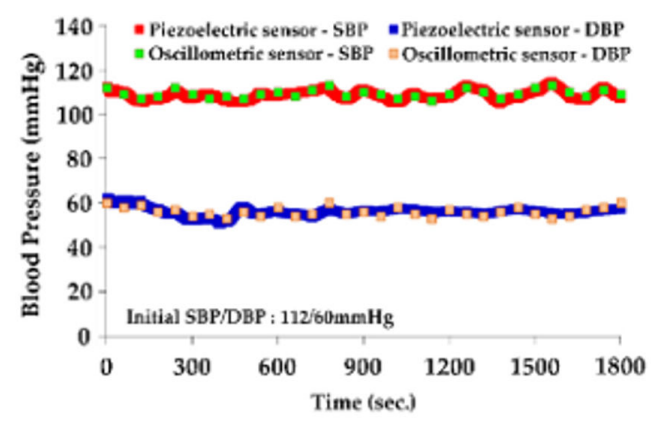

(b)

Figure 11. (a) PPW signals from the radial artery for $30 \mathrm{~min}$ measurement. (b) Accuracy evaluation for a piezoelectric-base system for 30 min measurement, compared to an oscillometric sensor (Subject 1). 
Table 1. Accuracy evaluation for our system.

\begin{tabular}{|c|c|c|c|c|c|c|}
\hline Subject & Male & Age & $\begin{array}{l}\text { Height } \\
\text { (cm) }\end{array}$ & $\begin{array}{l}\text { Weight } \\
\text { (kg) }\end{array}$ & $\begin{array}{c}\text { SBP Error } \\
(\mathrm{MAE} \pm \mathrm{SD} \mathrm{mmHg})\end{array}$ & $\begin{array}{c}\text { DBP Error } \\
(\mathrm{MAE} \pm \mathrm{SD} \mathrm{mmHg})\end{array}$ \\
\hline 1 & Male & 58 & 180 & 90 & $1.34 \pm 0.07$ & $1.61 \pm 0.16$ \\
\hline 2 & Female & 57 & 150 & 68 & $1.51 \pm 0.13$ & $1.92 \pm 0.22$ \\
\hline 3 & Male & 60 & 170 & 80 & $1.46 \pm 0.17$ & $1.87 \pm 0.29$ \\
\hline 4 & Female & 36 & 155 & 66 & $1.62 \pm 0.21$ & $2.01 \pm 0.38$ \\
\hline 5 & Male & 31 & 168 & 65 & $1.24 \pm 0.27$ & $1.56 \pm 0.39$ \\
\hline 6 & Male & 36 & 175 & 90 & $1.44 \pm 0.33$ & $1.64 \pm 0.66$ \\
\hline 7 & Male & 27 & 175 & 68 & $1.47 \pm 0.22$ & $1.77 \pm 0.52$ \\
\hline 8 & Female & 27 & 156 & 55 & $1.7 \pm 0.42$ & $2.06 \pm 0.71$ \\
\hline 9 & Female & 24 & 160 & 90 & $1.39 \pm 0.35$ & $1.72 \pm 0.66$ \\
\hline 10 & Male & 28 & 180 & 110 & $1.58 \pm 0.44$ & $1.95 \pm 0.77$ \\
\hline 11 & Male & 36 & 176 & 105 & $1.22 \pm 0.21$ & $1.84 \pm 0.51$ \\
\hline 12 & Female & 27 & 163 & 55 & $1.38 \pm 0.19$ & $1.79 \pm 0.44$ \\
\hline 13 & Female & 27 & 164 & 61 & $1.66 \pm 0.47$ & $1.68 \pm 0.41$ \\
\hline 14 & Male & 27 & 168 & 68 & $1.53 \pm 0.38$ & $1.72 \pm 0.49$ \\
\hline 15 & Male & 27 & 165 & 79 & $1.47 \pm 0.24$ & $1.76 \pm 0.39$ \\
\hline 16 & Male & 27 & 168 & 72 & $1.51 \pm 0.26$ & $1.89 \pm 0.44$ \\
\hline 17 & Male & 27 & 170 & 68 & $1.42 \pm 0.33$ & $1.77 \pm 0.46$ \\
\hline 18 & Female & 59 & 156 & 71 & $1.45 \pm 0.22$ & $1.87 \pm 0.34$ \\
\hline 19 & Female & 54 & 154 & 50 & $1.38 \pm 0.28$ & $1.92 \pm 0.44$ \\
\hline 20 & Male & 23 & 168 & 66 & $1.54 \pm 0.36$ & $1.86 \pm 0.55$ \\
\hline 21 & Male & 27 & 163 & 62 & $1.58 \pm 0.32$ & $1.83 \pm 0.51$ \\
\hline 22 & Male & 23 & 178 & 72 & $1.56 \pm 0.28$ & $1.78 \pm 0.61$ \\
\hline 23 & Male & 20 & 173 & 68 & $1.33 \pm 0.15$ & $1.82 \pm 0.53$ \\
\hline 24 & Female & 21 & 160 & 58 & $1.49 \pm 0.24$ & $1.79 \pm 0.46$ \\
\hline 25 & Female & 27 & 160 & 54 & $1.62 \pm 0.33$ & $1.96 \pm 0.61$ \\
\hline 26 & Male & 20 & 175 & 53 & $1.84 \pm 0.37$ & $1.77 \pm 0.55$ \\
\hline 27 & Male & 20 & 174 & 105 & $1.58 \pm 0.41$ & $2.07 \pm 0.66$ \\
\hline 28 & Male & 23 & 183 & 71 & $1.77 \pm 0.55$ & $1.88 \pm 0.73$ \\
\hline 29 & Male & 20 & 170 & 85 & $1.67 \pm 0.41$ & $1.83 \pm 0.55$ \\
\hline 30 & Female & 20 & 155 & 61 & $1.89 \pm 0.43$ & $1.88 \pm 0.62$ \\
\hline Average & & & & & $1.52 \pm 0.30$ & $1.83 \pm 0.50$ \\
\hline
\end{tabular}

\section{Discussions}

\subsection{Measurement Strategy and Performance}

This study developed a wearable piezoelectric-based system that uses the sum of the initial blood pressure by an oscillometric method and pressure change by a piezoelectric sensor to obtain the beat-to-beat SBP and DBP. The continuous PPW signals extracted from the radial artery by a piezoelectric sensor directly reveals the arterial behaviors of expansion and contraction. The beat-to-beat pressure change was obtained by the pressure sensitivity of the piezoelectric sensor $(2 \mathrm{mV} / \mathrm{mmHg})$. In order to linearly convert the electrical signals to pressure signals by pressure sensitivity, the tested signals must stay within the pressure range of the sensor in the specification. The ranges of SBP and DBP are, respectively, from $70 / 40 \mathrm{mmHg}$ and 190/100 $\mathrm{mmHg}$, including categories of hypotension, normal blood pressure, and hypertension. We used a piezoelectric sensor with a pressure range of $-50-3000 \mathrm{mmHg}$ to ensure the system operation in the linear region; therefore, we believe that our method can obtain correct blood pressure values in different cardiovascular diseases by the combined evidence of sensor characteristics and experimental results. The experimental results indicated that the reliable MAE \pm SD for the SBP and DBP were $1.52 \pm 0.30 \mathrm{mmHg}$ and $1.83 \pm 0.50 \mathrm{mmHg}$, which agreed with the Association for the Advancement of Medical Instrumentation (AAMI) [21] and British Hypertension Society (BHS) [22] standard criteria of MAE within $5 \mathrm{mmHg}$ and SD within $\pm 8 \mathrm{mmHg}$. Therefore, this study demonstrates a novel measurement strategy that uses sensor properties to obtain accurate beat-to-beat blood pressure recording without multiple calibrations and complex regression analysis of the estimation model. 


\subsection{Accuracy Evaluation with Other Works}

A summary of recent studies on continuous blood pressure measurement is shown in Table 2. These works demonstrated the proposed technique with accuracy compared to cuff-sensor. Lazazzera et al. [9] presented a smartwatch of CareUpß)(Farasha Labs, Paris, France) for blood pressure estimation that uses the ECG and PPG physiological channels to calculate the PTT and estimate the blood pressure by a linear model. The experimental results indicated that the MAE \pm SD for SBP and DBP were validated by a sphygmomanometer, and obtained $1.52 \pm 9.45 \mathrm{mmHg}$ and $0.39 \pm 4.93 \mathrm{mmHg}$ on 44 subjects. Slapni`car et al. [10] created a blood pressure estimation model by PPG measurement and deep neural network. The results obtained MAE for SBP and DBP were, respectively, 9.43 and 6.88. Simjanoska et al. [23] provided a machine learning model to estimate the blood pressure from ECG recording on 51 different subjects and obtained MAE \pm SD for SBP and DBP were $7.72 \pm 10.22 \mathrm{mmHg}$ and $9.45 \pm 10.03 \mathrm{mmHg}$. Wang et al. [24] used PPW and PPG wave to calculate the PTT and established the blood pressure estimation model and resulted in an accuracy of $3.71 \pm 3.06 \mathrm{mmHg}$ for SBP and 5.44 $\pm 5.10 \mathrm{mmHg}$ for DBP. Liu et al. [25] used impedance plethysmography (IPG) technique to establish SBP model by linear regression and obtained the correlation coefficient of 0.7 with a cuff method. Liu et al. [17] provided a multiparameter fusion (MPF) estimation model that combined 21 features of PPW and computed the summation of 21 features of PPW for blood pressure estimation. The PPW-based mathematical model was used for further comparative analysis with the best PTT-based model and obtained the excellent estimation error of $0.7 \pm 7.78 \mathrm{mmHg}$ for SBP, and $0.83 \pm 5.45 \mathrm{mmHg}$ for DBP, compared to the PTT-based model of $1.33 \pm 0.37 \mathrm{mmHg}$ and $1.14 \pm 0.20 \mathrm{mmHg}$ for SBP and DBP.

Table 2. Recent works for blood pressure measurement.

\begin{tabular}{|c|c|c|c|c|c|}
\hline Author & Technique & $\begin{array}{l}\text { Statistic } \\
\text { Method }\end{array}$ & $\begin{array}{l}\text { SBP Error } \\
\text { (mmHg) }\end{array}$ & $\begin{array}{l}\text { DBP Error } \\
(\mathrm{mmHg})\end{array}$ & Ref. \\
\hline Lazazzera & $\mathrm{PTT}_{\mathrm{ECG}}$ PPG & $\mathrm{MAE} \pm \mathrm{SD}$ & $1.52 \pm 9.45$ & $0.39 \pm 4.93$ & [9] \\
\hline Simjanoska & $\mathrm{PTT}_{\mathrm{ECG}}$ & $\mathrm{MAE} \pm \mathrm{SD}$ & $7.72 \pm 10.22$ & $9.45 \pm 10.03$ & [23] \\
\hline Slapnicar & $\mathrm{PTT}_{\mathrm{PPG}}$ & MAE & 9.43 & 6.88 & [10] \\
\hline Wang & $\mathrm{PTT}_{\mathrm{PPW}}$ PPG & $\mathrm{MAE} \pm \mathrm{SD}$ & $3.71 \pm 3.06$ & $5.44 \pm 5.10$ & [24] \\
\hline Liu & $\mathrm{PTT}_{\mathrm{IPG}}$ & $\begin{array}{c}\text { Correlation } \\
\text { coefficient }\end{array}$ & 0.7 & - & [25] \\
\hline Liu & PPW & $\mathrm{MAE} \pm \mathrm{SD}$ & $0.70 \pm 7.78$ & $0.83 \pm 5.43$ & [17] \\
\hline Our work & PPW Pressure change & $\mathrm{MAE} \pm \mathrm{SD}$ & $1.52 \pm 0.30$ & $1.83 \pm 0.50$ & - \\
\hline
\end{tabular}

Compared to the above studies, we demonstrated a low-cost and accurate continuous beat-to-beat SBP/DBP detection system requiring fewer PPW features. Importantly, our system could obtain a qualified accuracy of MAE within $2 \mathrm{mmHg}$ and SD within $\pm 1 \mathrm{mmHg}$ without the need for multiple calibrations and complex regressions. We believe that our method is novel in that it is a step further than the previous studies.

\subsection{Limitation}

We used a straightforward method to convert voltage changes to pressure changes in PPW signals directly by piezoelectric properties of pressure sensitivity. However, stable PPW signals from the radial artery remain a significant issue that can also be affected by daily activities such as muscle changes caused by walking, eating, dressing, grabbing, etc. Our study used a solid piezoelectric sensor with an analog front-end to validate the direct estimation concept with the scope of measurement in the resting condition lasting for up to $30 \mathrm{~min}$. No doubt, the solid structure of the piezoelectric sensor could easily induce air gaps under extended use during daily activities. In our future work, we expect to (1) improve the design of flexible piezoelectric sensors, and optimized analog frontend construction may more appropriately address this important aspect in ambulatory blood pressure measurement, and 
(2) recruit more participants, especially patients with blood pressure problems, to make our method more reliable.

\section{Conclusions}

This paper develops a low-cost piezoelectric-based system to validate a straightforward computation for continuous beat-to-beat blood pressure measurement. The method uses the summation of initial blood pressure and pressure change without the need for calculating correlation and repeated calibrations. We estimated the continuous beat-to-beat blood pressure using the pressure sensitivity of the piezoelectric sensor to directly convert the voltage difference between adjacent systolic and diastolic feature points in PPW into pressure difference. The experimental results indicated our system produced a reliable accuracy of SBP (MAE $\pm \mathrm{SD} 1.52 \pm 0.30 \mathrm{mmHg})$ and $\mathrm{DBP}(\mathrm{MAE} \pm \mathrm{SD} 1.83 \pm 0.50 \mathrm{mmHg})$, which agreed with the AAMI of MAE within $5 \mathrm{mmHg}$ and SD within $\pm 8 \mathrm{mmHg}$. Overall, this study developed a low-cost wearable piezoelectric-based system using an intuitive measurement strategy with qualified accuracy. The new system is potentially suitable for continuous long-term blood pressure-monitoring applications.

Author Contributions: T.-W.W. conceived of the presented idea, carried out the experiment and wrote the manuscript. S.-F.L. supervised the project. All authors have read and agreed to the published version of the manuscript.

Funding: This research received no external funding.

Conflicts of Interest: The authors declare no conflict of interest.

\section{References}

1. Kitterman, J.A.; Phibbs, R.H.; Tooley, W.H. Catheterization of umbilical vessels in newborn infants. Pediatr. Clin. N. Am. 1970, 17, 895-912. [CrossRef]

2. Chen, S.; Ji, Z.; Wu, H.; Xu, Y. A Non-Invasive Continuous Blood Pressure Estimation Approach Based on Machine Learning. Sensors 2019, 19, 2585. [CrossRef] [PubMed]

3. Goli, S.; Jayanthi, T. Cuff less continuous non-invasive blood pressure measurement using pulse transit time measurement. Int. J. Recent Dev. Eng. Technol. 2014, 2, 16-86.

4. Choi, Y.; Zhang, Q.; Ko, S. Noninvasive cuffless blood pressure estimation using pulse transit time and Hilbert-Huang transform. Comput. Electr. Eng. 2013, 39, 103-111. [CrossRef]

5. Nye, E. The effect of blood pressure alteration on the pulse wave velocity. Br. Heart J. 1964, 26, 261. [CrossRef]

6. Gribbin, B.; Steptoe, A.; Sleight, P. Pulse wave velocity as a measure of blood pressure change. Psychophysiology 1976, 13, 86-90. [CrossRef]

7. Chen, W.; Kobayashi, T.; Ichikawa, S.; Takeuchi, Y.; Togawa, T. Continuous estimation of systolic blood pressure using the pulse arrival time and intermittent calibration. Med. Biol. Eng. Comput. 2000, 38, 569-574. [CrossRef]

8. Peter, L.; Noury, N.; Cerny, M. A review of methods for non-invasive and continuous blood pressure monitoring: Pulse transit time method is promising? Irbm 2014, 35, 271-282. [CrossRef]

9. Lazazzera, R.; Belhaj, Y.; Carrault, G. A New Wearable Device for Blood Pressure Estimation Using Photoplethysmogram. Sensors 2019, 19, 2557. [CrossRef]

10. Slapničar, G.; Mlakar, N.; Luštrek, M. Blood Pressure Estimation from Photoplethysmogram Using a Spectro-Temporal Deep Neural Network. Sensors 2019, 19, 3420. [CrossRef]

11. Sharma, M.; Barbosa, K.; Ho, V.; Griggs, D.; Ghirmai, T.; Krishnan, S.; Hsiai, T.; Chiao, J.-C.; Cao, H. Cuff-less and continuous blood pressure monitoring: A methodological review. Technologies 2017, 5, 21. [CrossRef]

12. Proença, J.; Muehlsteff, J.; Aubert, X.; Carvalho, P. Is pulse transit time a good indicator of blood pressure changes during short physical exercise in a young population? In Proceedings of the International Conference of the IEEE Engineering in Medicine and Biology Society, Buenos Aires, Argentina, 31 August-4 September 2010. 
13. Wong, M.Y.-M.; Poon, C.C.-Y.; Zhang, Y.-T. An evaluation of the cuffless blood pressure estimation based on pulse transit time technique: A half year study on normotensive subjects. Cardiovasc. Eng. 2009, 9, 32-38. [CrossRef] [PubMed]

14. Fung, P.; Dumont, G.; Ries, C.; Mott, C.; Ansermino, M. Continuous noninvasive blood pressure measurement by pulse transit time. In Proceedings of the International Conference of the IEEE Engineering in Medicine and Biology Society, San Francisco, CA, USA, 1-5 September 2004.

15. Mase, M.; Mattei, W.; Cucino, R.; Faes, L.; Nollo, G. Feasibility of cuff-free measurement of systolic and diastolic arterial blood pressure. J. Electrocarsiol. 2011, 44, 201-207. [CrossRef] [PubMed]

16. Kim, J.; Chou, E.F.; Le, J.; Wong, S.; Chu, M.; Khine, M. Soft Wearable Pressure Sensors for Beat-to-Beat Blood Pressure Monitoring. Adv. Healthc. Mater. 2019, 8, 1900109. [CrossRef] [PubMed]

17. Liu, Z.-D.; Liu, J.-K.; Wen, B.; He, Q.-Y.; Li, Y.; Miao, F. Cuffless Blood Pressure Estimation Using Pressure Pulse Wave Signals. Sensors 2018, 18, 4227. [CrossRef]

18. Kaisti, M.; Panula, T.; Leppänen, J.; Punkkinen, R.; Tadi, M.J.; Vasankari, T.; Jaakkola, S.; Kiviniemi, T.; Airaksinen, J.; Kostiainen, P. Clinical assessment of a non-invasive wearable MEMS pressure sensor array for monitoring of arterial pulse waveform, heart rate and detection of atrial fibrillation. NPJ Digit. Med. 2019, 2, 39. [CrossRef]

19. Spinelli, E.M.; Pallàs-Areny, R.; Mayosky, M.A. AC-coupled front-end for biopotential measurements. IEEE T. Bio Med. Eng. 2003, 50, 391-395. [CrossRef]

20. Çakar, H.I.; Kara, S.; Toker, O. Design of a Portable Electromyography Device for Back Herniated Patients. In Proceedings of the International Conference on Biomedical Electronics and Devices, Valencia, Spain, 20-23 January 2010.

21. White, W.B.; Berson, A.S.; Robbins, C.; Jamieson, M.J.; Prisant, L.M.; Roccella, E.; Sheps, S.G. National standard for measurement of resting and ambulatory blood pressures with automated sphygmomanometers. Hypertension 1993, 21, 504-509. [CrossRef]

22. O’Brien, E.; Petrie, J.; Littler, W.; de Swiet, M.; Padfield, P.L.; Altman, D.; Bland, M.; Coats, A.; Atkins, N. The British Hypertension Society protocol for the evaluation of blood pressure measuring devices. J. Hypertens 1993, 11, S43-S62.

23. Simjanoska, M.; Gjoreski, M.; Gams, M.; Madevska Bogdanova, A. Non-invasive blood pressure estimation from ECG using machine learning techniques. Sensors 2018, 18, 1160. [CrossRef]

24. Wang, Y.-J.; Chen, C.-H.; Sue, C.-Y.; Lu, W.-H.; Chiou, Y.-H. Estimation of Blood Pressure in the Radial Artery Using Strain-Based Pulse Wave and Photoplethysmography Sensors. Micromachines 2018, 9, 556. [CrossRef] [PubMed]

25. Liu, S.-H.; Cheng, D.-C.; Su, C.-H. A cuffless blood pressure measurement based on the impedance plethysmography technique. Sensors 2017, 17, 1176. [CrossRef] [PubMed] 